\title{
Alveolar Bone Loss, Tooth Loss and Oral Cancer Mortality in Older Patients: A Retrospective Cohort Study
}

This article was published in the following Dove Press journal: Clinical Interventions in Aging

\author{
Yifeng Qian ${ }^{1-3, *}$ \\ Huiting $\mathrm{Yu}^{4, *}$ \\ Weijun Yuan ${ }^{1-3, *}$ \\ Jiaqing $\mathrm{Wu}^{5}$ \\ Qingyu $X u^{5}$ \\ Nianrou $\mathrm{Mei}^{5}$ \\ Xudong Wang ${ }^{1-3}$ \\ Chunfang Wang ${ }^{4}$ \\ 'Department of Oral and \\ Craniomaxillofacial Surgery, Shanghai \\ Ninth People's Hospital, College of \\ Stomatology, Shanghai JiaoTong \\ University School of Medicine, Shanghai, \\ People's Republic of China; ${ }^{2}$ National \\ Clinical Research Center for Oral \\ Diseases, Shanghai, People's Republic of \\ China; ${ }^{3}$ Shanghai Key Laboratory of \\ Stomatology \& Shanghai Research \\ Institute of Stomatology, Shanghai, \\ People's Republic of China; ${ }^{4}$ Shanghai \\ Municipal Center for Disease Control \\ and Prevention, Shanghai, People's \\ Republic of China; ${ }^{5}$ College of \\ Stomatology, Shanghai JiaoTong \\ University School of Medicine, Shanghai, \\ People's Republic of China
}

*These authors contributed equally to this work
Correspondence: Xudong Wang; Chunfang Wang Email xudongwang70@hotmail.com; qyf208@I63.com
Purpose: There has been growing interest in the association between periodontitis and systemic disease. In recent years, however, inconsistent results have also been found by case-control studies for the role of periodontitis in the development of oral cancer. This study aimed to examine whether periodontitis was an independent risk factor for oral cancer with a $\geq 75$-year age group cohort.

Materials and Methods: Between January 2010 and December 2014, 1385 patients aged $\geq 75$ years who underwent radiographic examination were included in this retrospective cohort study. We collected demographic information and comorbid health conditions from local health authorities. Participants were followed up until either the occurrence of mortality, or the end of the study on December 31, 2018. Cox proportional hazards regression and competing risk hazard models were used to examine the association between periodontitis and oral cancer mortality.

Results: Periodontitis and loss of teeth were significantly associated with oral cancer mortality. Compared to oral cancer mortality in healthy subjects, the HR and $95 \% \mathrm{CI}$ in patients with mild, moderate, and severe periodontitis were 4.46 (0.94-21.06), 5.16 (1.14-23.39), and 6.65 (1.51-29.36), respectively. The HR (95\% CI) was 1.05 (1.01-1.09) for tooth loss after controlling for potential confounding factors. All the increases in risk persisted in patients aged $\geq 80$ years.

Conclusion: The present study provides substantial evidence that poor periodontal health is associated with oral cancer mortality. It is necessary to underline the importance of considering periodontitis in the prevention of oral cancer, particularly in the older patients.

Keywords: periodontitis, alveolar bone loss, tooth loss, oral cancer, prevention

\section{Introduction}

Oral cancer is a common malignant tumor in the head and neck region, and oropharyngeal cancer ranks sixth among all malignant tumors worldwide. In some high-incidence areas, new cases of oral cancer account for $25 \%$ of malignant tumors in males every year. ${ }^{1}$ The treatment of oral cancer often leads to the loss of important functions, including loss of clarity of speech, dysphagia, eating disorders, and changes in facial appearance, which has a serious impact on the quality of life of patients. ${ }^{2}$ Therefore, the prevention of oral cancer is particularly important.

Periodontal disease (PD) is a common and destructive disease of the oral cavity, which can lead to tooth loss. The development of PD is a complex process that occurs over a long period of time due to bacterial infection and inflammation, 
subsequently involving periodontal fibers and alveolar bone, the supporting structures of teeth. ${ }^{3}$ Some studies have suggested that $\mathrm{PD}$ independently contributed to increased malondialdehyde (MAA) and asymmetric dimethylarginine (ADMA) levels in saliva and serum. ${ }^{4,5}$ The increase of MAA and ADMA levels are associated with endothelial carotid damage in patients with atherosclerosis and are independent risk factors for cardiovascular disease mortality. ${ }^{6,7}$ The presence of PD was also found to negatively influence serum vitamin D levels. ${ }^{8}$ The vitamin D receptor presents in the cells of the immune system can protect the endothelium of periodontal tissues and by reducing the production of $\mathrm{B}$ and $\mathrm{T}$ lymphocytes which are released following the insult of periodontopathogenic bacteria. ${ }^{9,10}$

Poor oral health or hygiene, as indicators of PD, has been found to be risk factor, independent from other significant risk factors including smoking and alcohol consumption, in cancer development. ${ }^{11-16}$ In particular, the severity and prevalence of periodontitis have been found to be increased in patients with oral cancer in recent years. ${ }^{17,18}$ However, to date, the role of PD in the development of oral cancer is still controversial. Additionally, almost all the existing studies were case control studies, information on the above association is limited from other study design. ${ }^{19-22}$

We conducted a retrospective cohort study in a cohort of $\geq 75$-year age to examine the relationship between periodontitis and oral cancer mortality. The null hypothesis of the current study was that periodontitis is an independent risk factor for oral cancer mortality in older patients.

\section{Methods}

From January 1, 2010 to December 31, 2014, a total of 30,169 permanent residents aged $\geq 75$ years received periodontal treatment at Shanghai Ninth People's Hospital, the largest dental hospital in Shanghai. We inquired about the imaging data on a case-by-case basis to obtain the objective oral health status of the patients. Finally, 1385 patients who underwent panoramic radiography were included in our cohort. All the patients in our study were followed up until December 31, 2018. Subjects were censored at the end of follow-up or mortality from other causes other than oral cancer.

In the current study, measurements of alveolar bone loss were made from the cementoenamel junction to the tooth apex (total root length) and from the marginal bone crest to the tooth apex (total bone height). The proportion of the remaining bone height of the teeth was calculated from the total root length and total bone height. Participants were subsequently allocated to four groups based on the quartile of the above proportion: healthy ( $\geq 51 \%$ remaining bone), mild periodontitis ( $43 \%$ to $51 \%$ remaining bone), moderate periodontitis (26-43\% remaining bone), and severe periodontitis $(<26 \%$ remaining bone). Dentures (complete/partial) and complete implant bridge, in either jaw were not included under the scope of the residual teeth. The maximum number of teeth was 28 as the third molars were excluded.

Demographic and mortality information for the subjects in our cohort was updated at annual intervals from the electronic health records and mortality database in Shanghai Municipal Center for Disease Control and Prevention, which covers all the fixed population in Shanghai. We also collected the comorbid health conditions from the Shanghai Health Information Center, where outpatient, hospital admission and discharge records for all the hospitals in Shanghai are stored. All the diseases were coded using the International Classification of Diseases, Revision 10 (ICD 10). We defined "oral cancer" mortality as codes C00-08 (except C05.1, C05.2, and C01) as the underlying causes of death on the death certificate. Body mass index (BMI) was calculated as weight (in kilograms) divided by stature (in meters) squared $\left(\mathrm{kg} / \mathrm{m}^{2}\right)$. Subjects with a BMI $<25$ were classified as normal and those with a BMI $\geq 25$ were classified as overweight. The education levels of the participants were categorized into the following categories: $\leq 6$ years, 7-12 years, and university studies. Smoking status in our study was defined as nonsmoker, previous smoker, and current smoker. Drinking status was defined as never drink, drink sometimes and drink every day.

\section{Statistical Analysis}

The relevance of alveolar bone loss and tooth loss to the risk of oral cancer mortality was first fitted with a univariate Cox proportional hazards model. We then constructed a multivariable-adjusted model to control for the confounding effect of potential risk factors, including age, sex, BMI, education level, smoking, drinking, and the presence of comorbidities.

We also fitted competing risk hazard models based on Fine and Gray to identify risk factors for the cumulative incidence of specific events in the presence of competing risks. In these models, oral cancer mortality was set as the 
primary event of interest, and death from another cause was treated as a competing event.

All the reported $\mathrm{P}$ values in this study are based on two-sided tests at $\alpha=0.05$. The analysis was performed using R, version 3.4.3 (R Development Core Team 2010).

This study was approved by the Ethics Committee of the Shanghai Ninth People's Hospital (SH9H-2019-T36-2) and conducted in accordance with the Declaration of Helsinki. The patient data was maintained with confidentiality. Informed consent was exempted from the retrospective collection of patients' information.

\section{Results}

A total of 1385 subjects were followed up in this study. Among them, 40 patients died of oral cancer, while 317 cases died of other causes. Table 1 summarizes the baseline characteristics of all the participants and provides some indication regarding the factors that might be confounding factors in the main analysis. Subjects who died of oral cancer suffer from more severe alveolar bone loss and more missing teeth than survivors and subjects who died of other diseases. Additionally, subjects who died of oral cancer were more likely to be men, smokers and less educated, and less likely to have a history of hypertension and diabetes.

In this study, PD was defined by the alveolar bone loss, which was predictive of PD assessed using radiographs. Furthermore, there was a significant association between PD and oral cancer. Compared to oral cancer mortality in healthy subjects, the $\mathrm{HR}$ and $95 \% \mathrm{CI}$ in patients with mild, moderate, and severe periodontitis were 4.46 (0.94-21.06), 5.16 (1.14-23.39), and 6.65 (1.51-29.36), respectively. Also, HR (95\% CI) for the trend of alveolar bone loss was found to be 1.55 (1.12-2.14). Positive and substantial associations were also found between tooth loss and PD. The HR (95\% CI) was 1.05 (1.01-1.09) after controlling for potential confounding factors (Table 2).

We fitted competing risk hazard models to further examine the impact of PD on oral cancer mortality. As shown in Table 3, both alveolar bone loss and tooth loss were found to be associated with oral cancer mortality. The HR (95\% CI) for alveolar bone loss and tooth loss was $1.47(1.12-1.92)$ and 1.04 (1.00-1.08) respectively.

We performed a stratified analysis to detect possible susceptible subgroups for oral cancer mortality in the case of PD exposure. We found that alveolar bone loss significantly increased the risk of oral cancer death in subjects aged 80 years and above. The analysis of the impact of missing teeth also showed that missing teeth were more likely to increase the risk of death from oral cancer in people aged 80 and over (Figure 1).

\section{Discussion}

In this cohort study of patients with PD, a substantial increase in the risk of oral cancer mortality was recorded in older patients with severe alveolar bone loss compared with those who did not. A similar increase in risk was also noted for the number of teeth lost and oral cancer mortality. The increase in risk persisted in patients aged $\geq 80$ years. Given that both alveolar bone loss and tooth loss are markers of periodontitis, this information underlines the importance of considering periodontitis in the prevention of oral cancer, especially in older patients.

There has been growing interest in the association between periodontitis and oral cancer risks over the past decades. In our research, we recruited 1385 subjects aged $\geq 75$ years. The relatively large sample size in this age group offers us the possibility to explore the impact of periodontitis on oral cancer mortality with a cohort design. Our results are in accord with a recent study in India. $^{22}$ A meta-analysis, which included 5 case control studies, also reported a pooled OR adjusting for known risk factors (mostly smoking and alcohol consumption) for the association between periodontal disease and oral cancer of 3.53 (95\% CI: $1.52-8.23){ }^{23}$

To assess the robustness of the findings, we first fitted the univariate model to examine the effect of alveolar bone loss and tooth loss on oral cancer mortality. The confounding effect of age, sex, education level, BMI, smoking, drinking, hypertension, and diabetes were adjusted step by step. In sensitivity analyses, alveolar bone loss was considered as both a categorical variable and a ranked variable in the analysis. Finally, the potential relevance of the competing risk of non-oral cancer-related death was considered using the Fine and Gray competing risk regression modeling.

PD are difficult to measure and quantify in observational studies, as the assessment of PD requires several periodontal measurements. Some large cohort studies enrolling thousands of participants cannot conduct periodontal examinations on all subjects, and obtaining radiographs on all participants is not feasible in these studies. 
Table I Baseline Characteristics of Study Participants*

\begin{tabular}{|c|c|c|c|}
\hline & Survivors & Mortality of Oral Cancer & Mortality of Other Diseases \\
\hline Participants & $1028(74.22 \%)$ & $40(2.89 \%)$ & $317(22.89 \%)$ \\
\hline Residual alveolar bone percentage & $0.39(0.18)$ & $0.29(0.19)$ & $0.36(0.2 I)$ \\
\hline Loss of teeth & $\mid 3.48(8.21)$ & $16.83(9.11)$ & $14.38(9.13)$ \\
\hline Age & $80.31(3.70)$ & $82.04(5.42)$ & $82.05(4.47)$ \\
\hline \multicolumn{4}{|l|}{ Sex } \\
\hline Male & $488(47.50 \%)$ & $2 I(52.50)$ & I54(48.58) \\
\hline Female & $540(52.50 \%)$ & $19(47.50)$ & $163(51.42)$ \\
\hline \multicolumn{4}{|l|}{$\operatorname{BMI}\left(\mathrm{kg} / \mathrm{m}^{2}\right)$} \\
\hline$<25$ & $834(81.10 \%)$ & $32(80.00)$ & $27 I(85.49)$ \\
\hline$\geq 25$ & 194(18.90\%) & $8(20.00)$ & $46(|4.5|)$ \\
\hline \multicolumn{4}{|l|}{ Education levels(year) } \\
\hline$\leq 6$ & $406(50.24)$ & $21(55.26)$ & $102(36.56)$ \\
\hline $7-12$ & $326(40.35)$ & $14(36.84)$ & $|3|(46.95)$ \\
\hline$>12$ & $76(9.4 I)$ & $3(7.89)$ & $46(16.49)$ \\
\hline \multicolumn{4}{|l|}{ Smoking habits } \\
\hline Nonsmoker & $87 I(87.40 \%)$ & $27(77.14)$ & $250(84.46)$ \\
\hline Previous smoker & $102(10.20 \%)$ & $4(I 1.43)$ & $37(12.50)$ \\
\hline Current smoker & $24(2.40 \%)$ & $4(11.43)$ & $9(3.04)$ \\
\hline \multicolumn{4}{|l|}{ Drinking habits } \\
\hline Never drink & $816(81.90 \%)$ & $25(7 \mid .43)$ & $234(79.05)$ \\
\hline Drink sometimes & $164(16.50 \%)$ & $10(28.57)$ & $58(19.59)$ \\
\hline Drink everyday & $17(1.60 \%)$ & $0(0.00 \%)$ & $4(1.35)$ \\
\hline \multicolumn{4}{|l|}{ Hypertension } \\
\hline Without & $212(20.60 \%)$ & $22(55.00)$ & 107(33.75) \\
\hline With & $816(79.40 \%)$ & $18(45.00)$ & $210(66.25)$ \\
\hline \multicolumn{4}{|l|}{ Diabetes } \\
\hline Without & $947(92.10 \%)$ & $37(92.50)$ & $28 I(88.64)$ \\
\hline With & $81(7.90 \%)$ & $3(7.50)$ & $36(11.36)$ \\
\hline
\end{tabular}

Notes: *Age, loss of teeth and proportion of remaining bone height are presented as mean (SD). The other variables are presented as numbers (\%).

Table 2 HR and 95\% Confidence Interval for Oral Cancer Mortality*

\begin{tabular}{|c|c|c|c|c|c|c|c|}
\hline & \multicolumn{5}{|c|}{ Quartile of Residual Alveolar Bone Percentage } & \multicolumn{2}{|l|}{ Loss of Teeth } \\
\hline & QI $(\geq 0.5 I)$ & $Q 2(0.43-0.5 I)$ & $Q 3(0.26-0.43)$ & $Q 4(<0.26)$ & \multirow{2}{*}{$\begin{array}{l}\mathrm{HR}(95 \% \mathrm{Cl}) \\
\text { for Trend }\end{array}$} & \multirow[t]{2}{*}{$\mathrm{HR}(95 \% \mathrm{Cl})$} & \multirow[t]{2}{*}{$\mathbf{P}$} \\
\hline & $\operatorname{HR}(95 \% \mathrm{Cl})$ & $\operatorname{HR}(95 \% \mathrm{Cl})$ & $\operatorname{HR}(95 \% \mathrm{Cl})$ & $\mathrm{HR}(95 \% \mathrm{Cl})$ & & & \\
\hline Model I & 1.00 & $3.42(0.94,12.43)$ & $4.12(1.16,14.60)$ & $5.23(1.51,18.06)$ & $I .5 \mathrm{I}(\mathrm{I} .12,2.02)$ & $1.05(1.01,1.08)$ & 0.019 \\
\hline Model 2 & 1.00 & $3.4 I(0.94, I 2.4 I)$ & $4.00(1.13,14.20)$ & $5.08(1.47,17.6)$ & $1.49(1.11,2.01)$ & $\mathrm{I} .04(\mathrm{I} .0 \mathrm{I}, \mathrm{I} .08)$ & 0.025 \\
\hline Model 3 & 1.00 & $4.14(0.88, \mid 9.5 I)$ & $5.3 I(I .17,24.01)$ & $7.00(1.59,30.88)$ & $1.60(1.15,2.21)$ & $1.05(1.01,1.09)$ & 0.019 \\
\hline Model 4 & 1.00 & $4.46(0.94,21.06)$ & $5.16(1.14,23.39)$ & $6.65(1.51,29.36)$ & $1.55(1.12,2.14)$ & $1.05(1.01,1.09)$ & 0.027 \\
\hline
\end{tabular}

Notes: *Model I: crude, no adjustment. Model 2: adjusting for age, sex, education level and BMI. Model 3: adjusting for age, sex, education level, BMI, smoking and drinking. Model 4: adjusting for age, sex, education level, BMI, smoking, drinking, hypertension and diabetes. QI: healthy; Q2: mild periodontitis; Q3: moderate periodontitis; Q4: severe periodontitis.

Alternatively, self-reported questions on oral health or the number of teeth were used as the crude measures of PD. ${ }^{24}$ In our study, all subjects underwent a panoramic radiographic examination. As a result, radiographic bone loss was considered as the defining criteria for the classification of periodontitis. Tooth loss is most commonly 
Table 3 HR and 95\% Confidence Interval of Competing Risk Models

\begin{tabular}{|l|l|l|l|}
\hline & $\begin{array}{l}\text { Fine-Gray } \\
\text { Test }\end{array}$ & $\mathbf{P}$ & $\begin{array}{l}\text { Adjusted HR } \\
\text { (95\% Cl)* }\end{array}$ \\
\hline $\begin{array}{l}\text { Severity of periodontitis } \\
\text { Loss of teeth }\end{array}$ & 8.45 & 0.038 & $1.47(\mathrm{I} .12, \mathrm{I} .92)$ \\
52.63 & 0.003 & $1.04(\mathrm{I} .00, \mathrm{I} .08)$ \\
\hline
\end{tabular}

Notes: *Adjusting for age, sex, education level, BMI, smoking, drinking, hypertension, and diabetes.

caused by dental caries and PD, but the percentage contribution from each condition depends on age and other factors. ${ }^{25}$ All the enrolled subjects in the current study were aged $\geq 75$ years, and tooth loss in this age group was more likely to be caused by chronic PD. Therefore, tooth loss in our study can be a better marker of PD compared with previous studies that covered populations of all age groups.

Evidence from multidisciplinary studies has lent support to the possibility of associations between periodontitis and cancer risk. Periodontal tissue infection can lead to systemic effects and an increase in circulating levels of inflammatory biomarkers such as C-reactive protein and interleukin-1 and $-6 .{ }^{26}$ Poor oral health can generate a potent carcinogen, nitrosamine. ${ }^{27}$ It has been reported that people with poor oral health have higher nitrosamine levels, which are driven by nitratereducing bacteria, in their oral cavities. ${ }^{28,29}$ In particular, oral bacteria including Porphyromonas gingivalis,
Fusobacterium nucleatum and Treponema denticola have been recognized as potential etiological agents of oral cancer and recently these pathogens have been found to dysregulate inflammatory mechanisms in oral cancer cells. $^{30,31}$

Certain limitations should be considered when interpreting the results of the present study. First, the dietary habits of the subjects were not considered in our study. Second, we did not perform clinical evaluation of PD. We used radiographic bone loss and tooth loss as the only objective indication of the severity of PD. Another limitation of this study was that we did not collect information regarding the treatment of $\mathrm{PD}$ in the current study. However, although the PD can be treated or managed to decrease further damage and grafting might be occasionally used to fill the bone loss, those with PD cannot be cured because the effects of past PD will generally manifest as attachment loss and bone loss. ${ }^{32}$

\section{Conclusion}

In summary, the study findings suggest that the risk of oral cancer mortality increases substantially in older patients with severe alveolar bone loss. Since this study supports the evidence that severity of PD is associated with oral cancer mortality, the control and prevention of PD should be emphasized, especially in older patients, as it could reduce the possible progression and risk of developing oral cancer.

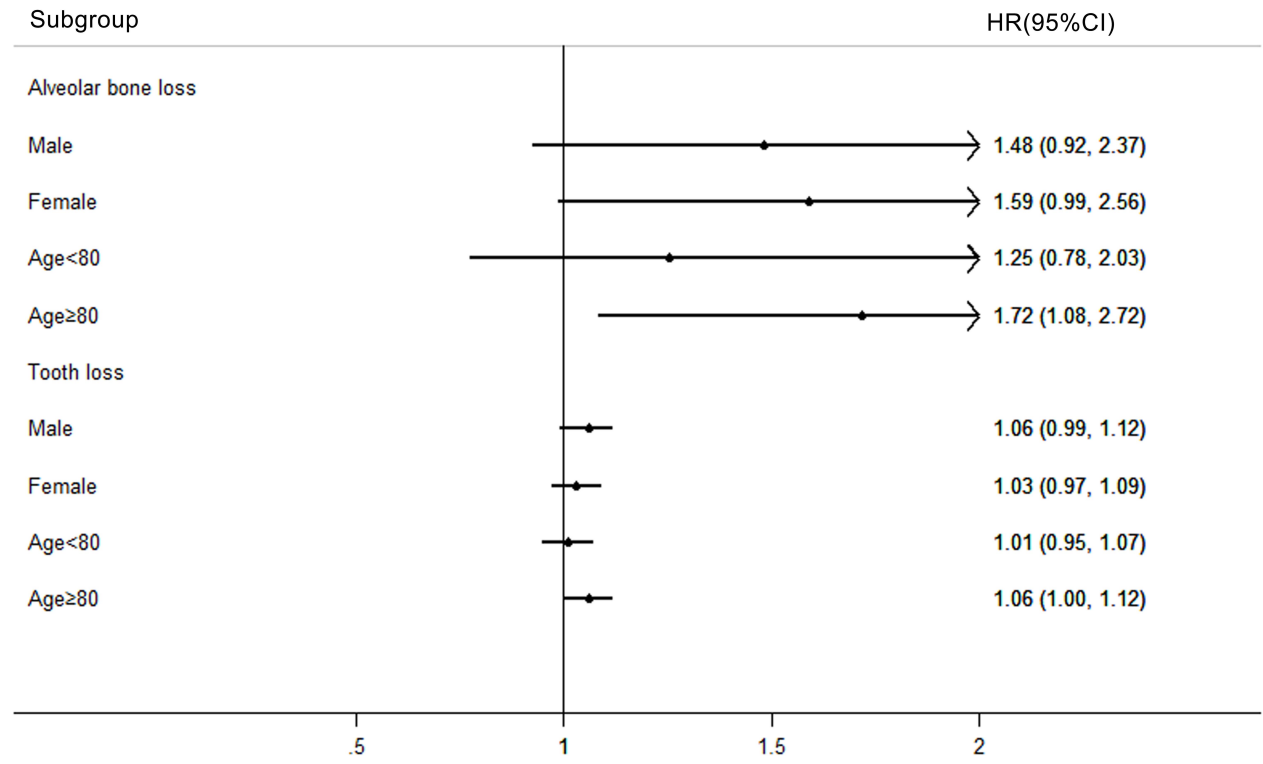

Figure I Stratified analysis by sex and age for oral cancer mortality. 


\section{Funding}

This research was supported by the National Natural Science Foundation of China (31971485). Shanghai's Top Priority Clinical Medicine Center (2017ZZ01011), Shanghai Municipal Key Clinical Specialty (shslczdzk01601) and Shanghai Clinical Research Center for Oral Diseases (19MC1910600).

\section{Disclosure}

The authors report no conflicts of interest in this work.

\section{References}

1. Warnakulasuriya S. Living with oral cancer: epidemiology with particular reference to prevalence and life-style changes that influence survival. Oral Oncol. 2010;46(6):407-410. doi:10.1016/j.oraloncology.2010.02.015

2. Rivera C. Essentials of oral cancer. Int J Clin Exp Pathol. 2015;8:11.

3. Sperr M, Kundi M, Tursic V, et al. Prevalence of comorbidities in periodontitis patients compared with the general Austrian population. J Periodontol. 2018;89(1):19-27. doi:10.1902/jop.2017.170333

4. Isola G, Alibrandi A, Curro M, et al. Evaluation of salivary and serum adma levels in patients with periodontal and cardiovascular disease as subclinical marker of cardiovascular risk. J Periodontol. 2020. doi:10.1002/JPER.19-0446

5. Isola G, Polizzi A, Santonocito S, et al. Expression of salivary and serum malondialdehyde and lipid profile of patients with periodontitis and coronary heart disease. Int J Mol Sci. 2019;20.

6. Hansson GK, Hermansson A. The immune system in atherosclerosis. Nat Immunol. 2011;12(3):204-212. doi:10.1038/ni.2001

7. Tomofuji T, Azuma T, Kusano H, et al. Oxidative damage of periodontal tissue in the rat periodontitis model: effects of a high-cholesterol diet. FEBS Lett. 2006;580(15):3601-3604. doi:10.1016/j.febslet.2006.05.041

8. Isola G, Alibrandi A, Rapisarda E, et al. Association of vitamin d in patients with periodontitis: a cross-sectional study. J Periodontal Res. 2020. doi: $10.1111 /$ jre. 12746

9. Stein SH, Livada R, Tipton DA. Re-evaluating the role of vitamin $\mathrm{d}$ in the periodontium. $J$ Periodontal Res. 2014;49(5):545-553. doi:10.1111/jre.12149

10. Hong Lai BD, Elliot K, Fishman GG, Jeffrey A, Brinker BW. Vitamin d deficiency is associated with the development of subclinical coronary artery disease in african americans with hiv infection: a preliminary study. J Invest Med. 2012;60:7.

11. Tezal M, Sullivan MA, Hyland A, et al. Chronic periodontitis and the incidence of head and neck squamous cell carcinoma. Cancer Epidemiol Biomarkers Prev. 2009;18(9):2406-2412. doi:10.1158/ 1055-9965.EPI-09-0334

12. Corbella S, Veronesi P, Galimberti V, et al. Is periodontitis a risk indicator for cancer? A meta-analysis. PLoS One. 2018;13(4): e0195683. doi:10.1371/journal.pone.0195683

13. Mine Tezal SGG, Genco RJ. Is periodontitis associated with oral neoplasms. J Periodontol. 2005;76:5.

14. Babic A, Poole EM, Terry KL, et al. Periodontal bone loss and risk of epithelial ovarian cancer. Cancer Causes Control. 2015;26 (6):941-947. doi:10.1007/s10552-015-0575-7

15. Mai X, LaMonte MJ, Hovey KM, et al. Periodontal disease severity and cancer risk in postmenopausal women: the buffalo osteoperio study. Cancer Causes Control. 2016;27(2):217-228. doi:10.1007/ s10552-015-0699-9
16. Chou SH, Tung YC, Wu LS, et al. Severity of chronic periodontitis and risk of gastrointestinal cancers: a population-based follow-up study from taiwan. Medicine (Baltimore). 2018;97(27):e11386. doi:10.1097/MD.0000000000011386

17. Moraes RC, Dias FL, Figueredo CM, et al. Association between chronic periodontitis and oral/oropharyngeal cancer. Braz Dent J. 2016;27(3):261-266. doi:10.1590/0103-6440201600754

18. Aral CA, Olcer SN, Aral K, et al. Oxidative stress, neutrophil elastase and igfbp7 levels in patients with oropharyngeal cancer and chronic periodontitis. Oral Dis. 2020. doi:10.1111/odi.13370

19. Tezal M, Sullivan MA, Reid ME, et al. Chronic periodontitis and the risk of tongue cancer. Arch Otolaryngol Head Neck Surg. 2007;133 (5):5. doi:10.1001/archotol.133.5.450

20. Divaris K, Olshan AF, Smith J, et al. Oral health and risk for head and neck squamous cell carcinoma: the carolina head and neck cancer study. Cancer Causes Control. 2010;21(4):567-575. doi:10.1007/ s10552-009-9486-9

21. Rosenquist K, Wennerberg J, Schildt E-B, et al. Oral status, oral infections and some lifestyle factors as risk factors for oral and oropharyngeal squamous cell carcinoma. A population-based case-control study in southern sweden. Acta Otolaryngol. 2005;125 (12):1327-1336. doi:10.1080/00016480510012273

22. Laprise C, Shahul HP, Madathil SA, et al. Periodontal diseases and risk of oral cancer in southern india: results from the hence life study. Int J Cancer. 2016;139(7):1512-1519. doi:10.1002/ijc.30201

23. Yao QW, Zhou DS, Peng HJ, et al. Association of periodontal disease with oral cancer: a meta-analysis. Tumour Biol. 2014;35 (7):7073-7077. doi:10.1007/s13277-014-1951-8

24. Michaud DS, Fu Z, Shi J, et al. Periodontal disease, tooth loss, and cancer risk. Epidemiol Rev. 2017;39(1):49-58. doi:10.1093/epirev/ mxx006

25. Renvert S, Persson RE, Persson GR. Tooth loss and periodontitis in older individuals: results from the swedish national study on aging and care. J Periodontol. 2013;84(8):1134-1144. doi:10.1902/ jop.2012.120378

26. Chrysanthakopoulos NA. Correlation between periodontal disease indices and lung cancer in greek adults a case - control study. Exp Oncol. 2016;38:5.

27. Abnet CC, Kamangar F, Dawsey SM, et al. Tooth loss is associated with increased risk of gastric non-cardia adenocarcinoma in a cohort of finnish smokers. Scand J Gastroenterol. 2009;40(6):681-687. doi:10.1080/00365520510015430

28. Yoon HS, Wen W, Long J, et al. Association of oral health with lung cancer risk in a low-income population of african americans and european americans in the southeastern united states. Lung Cancer. 2019;127:90-95. doi:10.1016/j.lungcan.2018.11.028

29. Nair J, Ohshima H, Nair UJ, et al. Endogenous formation of nitrosamines and oxidative DNA-damaging agents in tobacco users. Crit Rev Toxicol. 1996;26:13. doi:10.3109/10408449609017 928

30. Aral K, Milward MR, Gupta D, et al. Effects of porphyromonas gingivalis and fusobacterium nucleatum on inflammasomes and their regulators in $\mathrm{H} 400$ cells. Mol Oral Microbiol. 2020;35 (4):158-167. doi:10.1111/omi.12302

31. Zhang WL, Wang SS, Wang HF, et al. Who is who in oral cancer? Exp Cell Res. 2019;384(2):111634. doi:10.1016/j.yexcr.2019.11 1634

32. Michaud DS, Liu Y, Meyer M, et al. Periodontal disease, tooth loss, and cancer risk in male health professionals: a prospective cohort study. Lancet Oncol. 2008;9(6):550-558. doi:10.1016/S14702045(08)70106-2 


\section{Publish your work in this journal}

Clinical Interventions in Aging is an international, peer-reviewed journal focusing on evidence-based reports on the value or lack thereof of treatments intended to prevent or delay the onset of maladaptive correlates of aging in human beings. This journal is indexed on PubMed Central, MedLine, CAS, Scopus and the Elsevier
Bibliographic databases. The manuscript management system is completely online and includes a very quick and fair peer-review system, which is all easy to use. Visit http://www.dovepress.com/ testimonials.php to read real quotes from published authors.

Submit your manuscript here: https://www.dovepress.com/clinical-interventions-in-aging-journal 